Pacific Journal of Mathematic 


\title{
ON SECONDARY CHARACTERISTIC CLASSES IN COBORDISM THEORY
}

\author{
WeI-Lung TING
}

This paper introduces into cobordism theory a new notion borrowed from ordinary cohomology theory. Specifically, let $\xi$ be a $U(n)$-bundle over the $C W$-complex $X$. Let $E$ and $E_{0}$ be the total spaces of the associated bundles whose fibers are respectively the unit disc $E^{2 n} \subset C^{n}$ and the unit sphere $S^{2 n-1} \subset C^{n}$. The classifying map for $\xi$ gives rise to an element $U_{\xi} \in \Omega_{U}^{2 n}\left(E, E_{0}\right)$. One defines the Thom isomorphism $\varphi: \Omega_{U}^{q}(X) \rightarrow$ $\Omega_{U}^{q+2 n}\left(E, E_{0}\right)$ by $\varphi(x)=\left(p^{*} x\right) U_{\xi}$ and Euler class, $e(\xi)$ of $\xi$, by $e(\xi)=$ $p^{*-1} j^{*}\left(U_{\xi}\right)$. For each $\alpha=\left(\alpha_{1}, \alpha_{2}, \cdots\right)$, let $c f_{\alpha}(\xi) \in \Omega_{U}^{2|\alpha|}(X)$ be the Conner-Floyd Chern class of $\xi$, and $S_{\alpha}: \Omega_{U}^{q}(X, Y) \rightarrow$ $\Omega_{U}^{q+2|\alpha|}(X, Y)$ be the operation defined by Novikov. Then one has the relation, $\mathbf{S}_{\alpha}(e(\xi))=c f_{\alpha}(\xi) \cdot e(\xi)$. Now if $\xi$ is a bundle such that $e(\xi)=0$, then one can define a secondary characteristic class

$$
\Sigma_{\alpha}(\xi) \in \Omega_{U}^{*}(X) \bmod \left(S_{\alpha}-c f_{\alpha}(\xi)\right) \Omega_{U}^{*}(X)
$$

by using the above relation. The object of this paper is to study some of the properties of such secondary characteristic classes.

Secondary characteristic classes adapt particularly to the study of embedding and immersion problems. Massey and Peterson and Stein developed secondary characteristic classes in ordinary cohomology theory [4][7][8], and Lazarov has studied secondary characteristic classes in $K$-theory [3]. We hope the secondary characteristic classes given here, and the operations on cobordism, defined by Novikov, will have some applications on embedding and immersion problems.

The organization of the papers is as follows. In $\S 1$ we collect some results on cobordism theory and give the definition of secondary characteristic classes of cobordism theory. In $\S 2$ we give an example and carry out some computations of these characteristic classes.

1. Definition of secondary characteristic classes. Let $\xi$ be a $U(n)$-bundle over the $C W$-complex $X$. Let $E$ and $E_{0}$ be the total spaces of the associated bundles whose fibres are respectively the unit disc $E^{2 n} \subset C^{n}$ and the unit sphere $S^{2 n-1} \subset C^{n}$. Then the Thom complex is the quotient space $E / E_{0}$. In particular, if we take $\xi$ to be the universal $U(n)$-bundle over $B U(n)$, then the resulting Thom complex $M(\xi)$ is written $M U(n)$. The sequence of spaces 


$$
(M U(0), M U(1), \cdots, M U(n), \cdots)
$$

is a spectrum. Associated with this spectrum we have a cohomology functor, the groups of this cohomology functor are written $\Omega_{U I}(X, Y)$ and called complex cobordism groups. We know that $\Omega_{U}^{*}($.$) is a mul-$ tiplicative cohomology theory and $\Omega_{U}(P)$, where $P$ is a point, is a polynomial ring $Z\left[x_{1}, x_{2}, \cdots, x_{i}, \cdots\right]$ where $x_{i} \in \Omega_{U}^{-2 i}(P)$.

Next for each $U(n)$-bundle $\xi$ over $X$ the classifying map for $\xi$ induces a map

$$
\gamma: M(\xi) \longrightarrow M U(n) \text {. }
$$

The map $\gamma$ represents an element $U_{\xi} \in \Omega_{U}^{2 n}\left(E, E_{0}\right)$. We define the Thom isomorphism

$$
\varphi: \Omega_{U}^{q}(X) \longrightarrow \Omega_{U}^{q+2 n}\left(E, E_{0}\right)
$$

by $\varphi(x)=\left(p^{*} x\right) U_{\xi}$.

Now we need the following known theorems:

THEOREM 1 (Conner-Floyd) [1]. To each $\xi$ over $X$ and each $\alpha=\left(\alpha_{1}, \alpha_{2}, \cdots\right)$ we can assign classes $c f_{\alpha}(\xi) \in \Omega_{U}^{2|\alpha|}(X)$, called the Conner-Floyd classes, with the following properties:

(i) $c f_{0}(\xi)=1$;

(ii) $c f_{\alpha}\left(g^{*} \xi\right)=g^{*} c f_{\alpha}(\xi)$;

(iii) Whitney sum formula $c f_{\alpha}(\xi \oplus \eta)=\sum_{\beta+\gamma=\alpha}\left(c f_{\beta} \xi\right)\left(c f_{\gamma} \eta\right)$;

(iv) Let $\xi$ be a U(1)-bundle over $X$, classified by a map $X \longrightarrow$ $B U(1)$, and let the composite $X \stackrel{f}{\longrightarrow} B U(1) \longrightarrow M U(1)$ represent the element $w \in \Omega_{U}^{2}(X)$. Then $c f_{1}(\xi)=w$.

TheOREM 2 (Novikov) [1]. For each $\alpha=\left(\alpha_{1}, \alpha_{2}, \cdots\right)$ there exists an operation

$$
S_{\alpha}: \Omega_{U}^{q}(X, Y) \longrightarrow \Omega_{U}^{q+2|\alpha|}(X, Y)
$$

with the following properties:

(i) $S_{0}=1$;

(ii) $S_{\alpha} f^{*}=f^{*} S_{\alpha}$;

(iii) $S_{\alpha}$ is stable: $S_{\alpha} \delta=\delta S_{\alpha}$;

(iv) Cartan formula

$$
S_{\alpha}(x y)=\sum_{\beta+\gamma=\alpha}\left(S_{\beta} x\right)\left(S_{\gamma} y\right)
$$

(vi) If $w \in \operatorname{Map}(X, M U(1)) \subset \Omega_{U}^{2}(X)$ then $S_{(k)}(w)=w^{k+1}$, and

$$
S_{\alpha}(w)=0 \text { if } \alpha \neq(k) \text {; }
$$

(vii) Suppose that $\xi$ is an $U(n)$-bundle over $X$ then we have 


$$
c f_{\alpha}(\xi)=\varphi^{-1} S_{\alpha} \varphi(1)
$$

where $\varphi$ is the Thom isomorphism for $\Omega_{U}^{*}$.

DEFINITION 3. The Euler class of a $U(n)$-bundle $\xi$ over $X$, denoted $e(\xi)$, is $p^{*-1} j^{*}\left(U_{\xi}\right)$, where $j^{*}: \Omega_{U}^{i}\left(E_{1}, E_{0}\right) \longrightarrow \Omega_{U}^{i}(E)$ is induced by the inclusion $j: E \longrightarrow\left(E, E_{0}\right)$, and the isomorphism $p^{*}: \Omega_{U}^{i}(X) \longrightarrow$ $\Omega_{U}^{i}(E)$ is induced by the projection $p: E \longrightarrow X$.

The following propositions are not difficult to prove:

Proposition 4. If $\xi$ is a trivial, then $e(\xi)=0$.

Proposition 5. For the Euler class, the relation

$$
e(\xi \oplus \eta)=e(\xi) e(\eta)
$$

holds.

Proposition 6. If a $U(n)$-bundle has an nonzero cross section, then $e(\xi)=0$.

From Theorem 2 we have $c f_{\alpha}(\xi)=\varphi^{-1} S_{\alpha} \varphi(1)$ so that

$$
S_{\alpha} U_{\xi}=\varphi c f_{\alpha}(\xi)=p^{*}\left(c f_{\alpha}(\xi)\right) U_{\xi} .
$$

Therefore we have $S_{\alpha} e(\xi)=c f_{\alpha}(\xi) e(\xi)$.

Now let $\xi$ be a bundle such that $e(\xi)=0$, then the long exact sequence for $\left(E, E_{0}\right)$ breaks up into short exact sequences.

$0 \longrightarrow \Omega_{U}^{i}(X) \longrightarrow \Omega_{U}^{i}\left(E_{0}\right) \stackrel{\delta}{\longrightarrow} \Omega_{U}^{i+1}\left(E, E_{0}\right) \longrightarrow 0$. Let $a_{\xi} \in \Omega_{U}^{2 n-1}\left(E_{0}\right)$ such that $\delta\left(\alpha_{\xi}\right)=U_{\xi}$. Then every element in $\Omega_{U}^{i}\left(E_{0}\right)$ can be written uniquely as $x a_{\xi}+y$ where $x \in \Omega_{U}^{i-(2 n-1)}(X)$ and $y \in \Omega_{U}^{i}(X)$. In particular, write $S_{\alpha}(a)=x a_{\xi}+y$. Then we apply $\delta$ and find that $x=c f_{\alpha}(\xi)$. If $a^{1}$ is another element with $\left(a^{1}\right)=U_{\xi}$, then $S_{\alpha}\left(a^{1}\right)=c f_{\alpha}(\xi) a^{1}+y^{1}$. Then $y-y^{1} \in\left(S_{\alpha}=c f_{\alpha}(\xi)\right) \Omega_{U}^{2 n-1}(X)$. Thus we can define a natural transformation $\Sigma_{\alpha}$, from $U(n)$-bundle whose $e(\xi)$-class vanishes, to a natural quotient of $\Omega_{U}^{*}$. If $\xi$ is a such bundle $\Sigma_{\alpha}(\xi)$ takes values in $\Omega_{U}^{*}(X)$ $\bmod \left(S_{\alpha}-c f_{\alpha}(\xi)\right) \Omega_{U}^{*}(X)$ and is the coset of $y$.

The following property can be easily proved:

Proposition 7. If $\xi$ has a nonzero cross section then $\Sigma_{\alpha}(\xi)=0$.

2. Example. Consider $U(n+1)$ as a principal $U(n)$-bundle over $S^{2 n+1}$ for $n>1$. Let $\xi$ be the associated complex vector bundle. Then the sphere bundle is the complex Stiefel manifold $U(n+1) / U(n-1)$. Since $\Omega_{U}^{2 n}\left(S^{2 n+1}\right)=0$, then $\Sigma_{\alpha}(\xi)$ is defined. 
Let $t_{n}$ be the Thom space of $S^{2 n+1}$ with respect to $\xi$, we have the short exact sequence

$$
0 \longrightarrow \Omega_{U}^{2 n-1}\left(S^{2 n+1}\right) \longrightarrow \Omega_{U}^{2 n-1}(U(n+1) / U(n-1)) \longrightarrow \Omega_{U}^{2 n}\left(t_{n}\right) \longrightarrow 0 .
$$

Since $H^{*}(U(n+1) / U(n-1))=\Lambda\left[\gamma_{2 n-1}, \gamma_{2 n+1}\right]$ be the exterior algebra generated by $\gamma_{2 n-1}$ and $\gamma_{2 n+1}$ of dimensions $2 n-1,2 n+1$ respectively. Therefore by [2] we have $\Omega_{U}^{*}(U(n+1) / U(n-1)) \Lambda\left[\gamma_{2 n-1}, \gamma_{2 n+1}\right] \otimes \Omega_{U}^{*}(P)$. Let $\quad \widetilde{\gamma}_{2 n-1} \in \Omega_{U}^{*}(U(n+1) / U(n-1)), \widetilde{\gamma}_{2 n+1} \in \Omega_{U}^{*}(U(n+1) / U(n-1))$ such that $\mu_{z}\left(\widetilde{\gamma}_{2 n-1}\right)=\gamma_{2 n-1}, \mu_{z}\left(\widetilde{\gamma}_{2 n+1}\right)=\gamma_{2 n+1}$, where $\mu_{z}: \Omega_{U}^{*} \longrightarrow H^{*}(, Z)$ is the map defined by the Thom class (see [2] for definition), the group $\Omega_{U}^{2 n-1}(U(n+1) / U(n-1))$ is $Z+Z$ with generators $\widetilde{\gamma}_{2 n-1}$ and $\widetilde{\gamma}_{2 n+1}\left[C P^{1}\right]$ where $\left[C P^{1}\right] \in \Omega_{U}^{-2}(P)$ is a generator of $\Omega_{U}^{*}(P)$. The group $\Omega_{U}^{2 n-1}\left(S^{2 n+1}\right)$ is infinite cyclic with generator $\widetilde{\gamma}_{2 n+1}\left[C P^{1}\right]$, and so $\delta\left(\widetilde{\gamma}_{2 n-1}\right)= \pm U_{\xi}$. We know that $S_{(1)} \widetilde{\gamma}_{2 n-1}=c f_{(1)} \widetilde{\gamma}_{2 n-1}+b \widetilde{\gamma}_{2 n+1}$ where $\pm b \widetilde{\gamma}_{2 n+1}$ represents $\Sigma_{(1)}(\xi)$. Since $\Omega_{U}^{*}(U(n+1) / U(n-1))$ injects into $\Omega_{U}^{*}(U(n+1))$, we can compute it in $\Omega_{U}^{*}(U(n+1))$. Now by using the notation of $[9, \mathrm{p} .40]$ we have the monomorphism

$$
\mu^{*}: \Omega_{U}^{*}(U(n+1)) \longrightarrow \Omega_{U}^{*}\left(Q_{n+1} \times U(n)\right) .
$$

By induction, we can determine $S_{(1)}$ if we know $S_{(1)}$ in $Q_{n+1}$ and its behavior under cross products. By [9] we have $Q_{n+1}=S C P^{n} V S^{1}$ and since $S_{(1)}$ commutes with the suspension map

$$
s: \Omega_{U}^{i}\left(C P^{n}\right) \longrightarrow \Omega_{U}^{i+1}\left(S C P^{n}\right),
$$

so we need only know $S_{(1)}$ in $\Omega_{U}^{*}\left(C P^{n}\right)$. By [2, p. 52] we know that $\Omega_{U}^{*}\left(C P^{n}\right)$ is a free $\Omega_{U}^{*}(P)$-module with basis $1, w_{n}, \cdots,\left(w_{n}\right)^{n}$ where $w_{n} \in \operatorname{Map}\left[C P^{n}, M U(1)\right] \subset \Omega_{U}^{2}\left(C P^{n}\right)$. Moreover, the inclusion

$$
\text { i: } C P^{n-1} \subset C P^{n}
$$

has $i^{*} w_{n}=w_{n-1}$. By Theorem 2 we have $S_{(1)}\left(w_{n}\right)^{j}=j\left(w_{n}\right)^{j+1}$, hence $S_{(1)} s\left(w_{n}\right)^{j}=s S_{(1)}\left(w_{n}\right)^{j}=s j\left(w_{n}\right)^{j+1}=j s\left(w_{n}\right)^{j+1}$, here $s\left(w_{n}\right)^{j}, s\left(w_{n}\right)^{j+1} \quad$ in $\Omega_{U}^{*}\left(S C P^{n}\right)$ are the images of $\left(w_{n}\right)^{i},\left(w_{n}\right)^{i+1}$ under the suspension map $s$ respectively. From above data and an argument, similar to [9, p. 53], we obtain $S_{(1)} \widetilde{\gamma}_{2 n-1}=(n-1) \widetilde{\gamma}_{2 n+1}$, hence $c f_{(1)}=0$ and $b=n-1$. Now we compute $\left(S_{(1)}-c f_{(1)}\right) \Omega_{U}^{2 n-1}\left(S^{2 n+1}\right)=S_{(1)} \Omega_{U}^{2 n-1}\left(S^{2 n+1}\right)$, which is generated by $S_{(1)}\left(\widetilde{\gamma}_{2 n+1}\left[C P^{1}\right]\right)$. By [5] we have $S_{(1)}\left(\widetilde{\gamma}_{2 n+1}\left[C P^{1}\right]\right)=2 \widetilde{\gamma}_{2 n+1}$. Therefore $\Sigma_{(1)}(\xi) \neq 0$ if $n-1 \neq 0 \bmod (2)$.

\section{REFERENCES}

1. J. F. Adams, S. P. Novikov's work on operations on complex cobordism, mimeographed notes, Chicago Univ., 1968.

2. P. E. Conner and E. E. Floyd, The relation of cobordism to $K$-theory, Lecture Notes in Math, Springer-Verlag, New York, 1966. 
3. C. Lazarov, Secondary characteristic classes in K-theory, Trans. Amer. Math. Soc. 136 (1969), 391-412.

4. W. S. Massey, On the embeddability of real projective spaces in Euclidean space, Pacific J. Math. 9 (1959), 783-789.

5. S. P. Novikov, The methods of Algebraic topology from the viewpoint of cobordism theory, Math USSR-Izvestija 1 (1967).

6. F, P. Peterson, Functional Cohomology operations, Trans. Amer. Math. Soc. 86 (1957). 197-211.

7. F. P. Peterson and N. Stein, Secondary cohomology operations, Amer. J. Math. 81 (1959), 281-305.

8. - Secondary characteristic classes, Ann. of Math. 76 (1962), 510-523.

9. N. E. Steenord, Cohomology operations, Ann. of Math. Study 50, Princeton, 1962.

Received August 29, 1969. Supported by the Research Foundation, State University of New York.

State University of NeW York

College of Arts \& Science at Plattsburgh 



\section{PACIFIC JOURNAL OF MATHEMATICS}

\section{EDITORS}

H. SAMELSON

Stanford University

Stanford, California 94305

\section{RichaRd PIERCe}

University of Washington

Seattle, Washington 98105
J. DUGUNDJI

Department of Mathematics

University of Southern California

Los Angeles, California 90007

RICHARD ARENS

University of California

Los Angeles, California 90024

\section{ASSOCIATE EDITORS}

E. F. BECKENBACH

B. H. NeUManN

F. WOLF

K. YosHIDA

\section{SUPPORTING INSTITUTIONS}

UNIVERSITY OF BRITISH COLUMBIA CALIFORNIA INSTITUTE OF TECHNOLOGY UNIVERSITY OF CALIFORNIA MONTANA STATE UNIVERSITY

UNIVERSITY OF NEVADA

NEW MEXICO STATE UNIVERSITY

OREGON STATE UNIVERSITY

UNIVERSITY OF OREGON

OSAKA UNIVERSITY

UNIVERSITY OF SOUTHERN CALIFORNIA
STANFORD UNIVERSITY

UNIVERSITY OF TOKYO

UNIVERSITY OF UTAH

WASHINGTON STATE UNIVERSITY

UNIVERSITY OF WASHINGTON

${ }^{*} \quad{ }^{*} \quad{ }^{*}$
AMERICAN MATHEMATICAL SOCIETY
CHEVRON RESEARCH CORPORATION
TRW SYSTEMS
NAVAL WEAPONS CENTER




\section{Pacific Journal of Mathematics}

\section{Vol. 33, No. $1 \quad$ March, 1970}

Mir Maswood Ali, On some extremal simplexes ................... 1

Silvio Aurora, On normed rings with monotone multiplication........... 15

Silvio Aurora, Normed fields which extend normed rings of integers....... 21

John Kelly Beem, Indefinite Minkowski spaces..................... 29

T. F. Bridgland, Trajectory integrals of set valued functions ........... 43

Robert Jay Buck, A generalized Hausdorff dimension for functions and sets ......................................... 69

Vlastimil B. Dlab, A characterization of perfect rings . . . . . . . . . . . . 79

Edward Richard Fadell, Some examples in fixed point theory ............ 89

Michael Benton Freeman, Tangential Cauchy-Riemann equations and uniform approximation ............................. 101

Barry J. Gardner, Torsion classes and pure subgroups ................ 109

Vinod B. Goyal, Bounds for the solution of a certain class of nonlinear

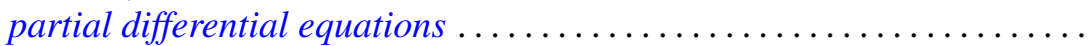

Fu Cheng Hsiang, On C, 1 summability factors of Fourier series at a given

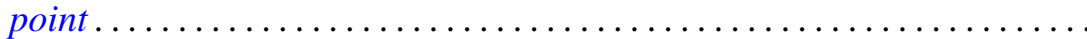

Lawrence Stanislaus Husch, Jr., Homotopy groups of PL-embedding

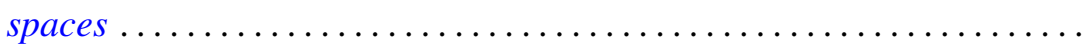

Daniel Ralph Lewis, Integration with respect to vector measures..........

Marion-Josephine Lim, $\mathscr{L}-2$ subspaces of Grassmann product spaces

Stephen J. Pierce, Orthogonal groups of positive definite multilinear functionals

W. J. Pugh and S. M. Shah, On the growth of entire functions of bounded index.

Siddani Bhaskara Rao and Ayyagari Ramachandra Rao, Existence of triconnected graphs with prescribed degrees . . .

Ralph Tyrrell Rockafellar, On the maximal monotonicity of subdifferential

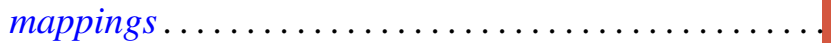

R. Shantaram, Convergence of a sequence of transformations of distribution functions. II ...............................

Julianne Souchek, Rings of analytic functions..............

Ted Joe Suffridge, The principle of subordination applied to functions of several variables...

Wei-lung Ting, On secondary characteristic classes in cobordism

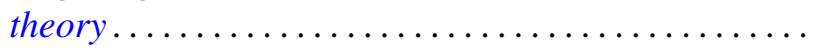

Pak-Ken Wong, Continuous complementors on $B^{*}$-algebras ...

Miyuki Yamada, On a regular semigroup in which the idempotents form a band. 